\title{
Diseño de una articulación de rodilla basada en un mecanismo policéntrico de cuatro barras cruzado tipo Chebyshev
}

\author{
Serrano-Molina, Hugo L. ${ }^{\mathrm{a}, 1, *}$, Lozano-Hernández, Yair ${ }^{\mathrm{b}}$, Galván-Guerra, Rosalba $^{\mathrm{c}}$ \\ ${ }^{a}$ Unidad Profesional Interdisciplinaria en Ingeniería y Tecnologías Avanzadas, Instituto Politécnico Nacional, CDMX, México. \\ ${ }^{b}$ Escuela Superior de Ingeniería Mecanica y Eléctrica unidad Zacatenco, Instituto Politécnico Nacional, CDMX, México. \\ ${ }^{c}$ Unidad Profesional Interdisciplinaria de Ingeniería campus Hidalgo, Instituto Politécnico Nacional, Hidalgo, México.
}

\section{Resumen}

En este trabajo se presentan los resultados del diseño de una articulación de rodilla para prótesis, exoesquelétos y robots bípedos. Para tal diseño, se hace uso de un mecanismo de cuatro barras, el cual es resultado de la síntesis geométrica para mecanismos planares. La propuesta desarrollada tiene ventajas anatómicas para reproducir el movimiento de la rodilla sobre el eje sagital. En el ciclo de marcha, se plantea una configuración de barras cruzadas, que mimetiza el desempeño de los ligamentos cruzados de la rodilla. De acuerdo a los resultados obtenidos, fue posible construir un prototipo con las cualidades mínimas de una prótesis transfemoral activa para realizar el ciclo completo de marcha, que sea adecúa a los parámetros de locomoción en adultos.

Palabras Clave: Prótesis transfemoral activa, Mecanismo 4-barras, Mecánismo Chebyshev, Síntesis geométrica, Dispositivos biomédicos.

\section{Introducción}

En la actualudad, la importancia de desarrollar prótesis para amputaciones arriba de la rodilla (trastibial), radica en la frecuencia de casos que se registran en la República Mexicana, con una prevalencia de hasta el $33 \%$ del universo de casos de amputaciones. Por lo anterior, existen diversos estudios orientados a resolver el problema de restitución de la porción pérdida y a su vez, generar el ciclo de marcha similar al de una extremidad humana sana. Los estudios enfocados en mejorar la eficiencia de las tareas de la rodilla se pueden consultar en (Gini et al., 2007; Narang et al., 2016). Por otro lado, en (Herr and Wilkenfeld, 2003; Martinez et al., 2014) se han reportado avances relevantes respecto al tipo de actuador que gobierna el giro de la rodilla prostética. Las estructuras policéntricas han demostrado una alternativa adecuada para representar las condiciones dinámicas de una rodilla humana (Radcliffe and Deg, 2003; Miller and Childress, 2005). Con la finalidad de imitar la habilidad en la que una rodilla humana se mueve durante el ciclo de marcha, en (Ranzani, 2014) proponen un controlador por impedancia adaptable, que requiere estimar parámetros musculares de la porción perdida; por otro lado, en (Poliakov et al., 2013) investigan un sistema de control que tiene como

\footnotetext{
*Autor en correspondencia: hserrano9800@alumno.ipn.mx

Correos electrónicos: hserrano9800@alumno.ipn.mx (Serrano-Molina, Hugo L. ), ylozanoh@ipn.mx (Lozano-Hernández, Yair ), rgalvang@ipn.mx (Galván-Guerra, Rosalba)
}

base la optimización dinámica de la posición de un mecanismo de cuatro barras, que es activado mediante la rigidez variable de un amortiguador. Según la expectativa del Instituto Nacional de Estadistica y Geografia, las enfermedades metabólicas y accidentes serán las principales causas que agravaran el problema de amputaciones de una extremidad inferior, durante los próximos diez años, por ello es imperativo perfeccionar los dispositivos prostéticos para rehabilitar el ciclo de marcha. En este artículo se propone el uso de un mecanismo policéntrico como articulación prostética de rodilla configurado a partir de la disposición geométrica de la misma en relación a los ligamentos cruzados, mostrados en la Figura 1; siendo posible aplicar una métodología de estudio de dinámica inversa para un mecanismo cruzado de cuatro barras. Mediante de la resolución cinemática y con los datos de posición, velocidad y aceleración, es posible calcular el torque necesario para mover la articulación. La configuración del mecanismo para la rodilla, está hecha con base en el mecanismo de cuatro barras cruzado tipo Chebyshev (Kempe, 1877; Xydas, 2014). El dimensionamiento de los eslabones se realiza tomando en cuenta las consideraciones descritas en (Kempe, 1875).

La propuesta de valor de este trabajo, radica en aprovechar las áreas de oportunidad del estado del arte, evitando el uso de actuadores que presentan importantes no linealidades como los amortiguadores magnetorreológicos, identificar los aspectos biomiméticos esenciales de la rodilla humana y ampliar los estados finitos, haciendo que tengan una relación directa con las 
fases del ciclo de marcha humana sana. Los principales resultados se obtuvieron mediante la simulación en Matlab del torque que debe imprimir un actuador lineal para movilizar una articulación prostética, comparable con el torque natural medido en una rodilla humana sana.

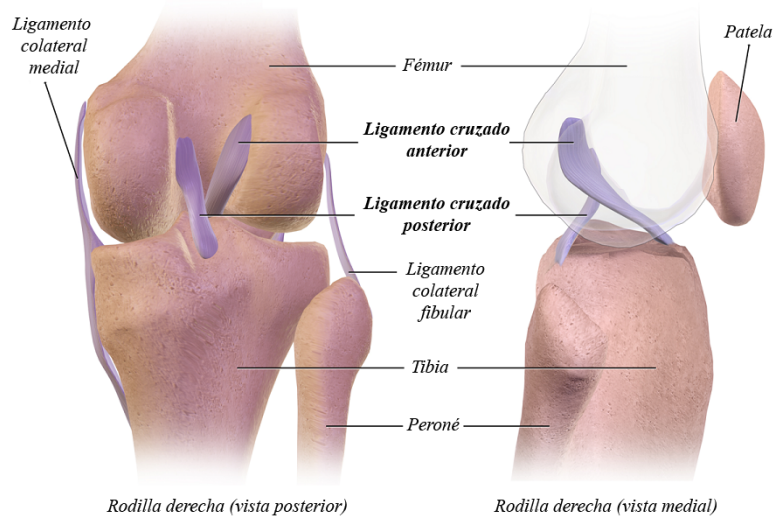

Figura 1: Ubicación de los ligamentos en una rodilla humana sana (Hamill and Knutzen, 2006)

El trabajo se organiza de la siguiente manera: en la sección 2 se presenta el procedimiento matemático para delinear las condiciones para una rodilla mecánica activa; en la sección 3 se muestra un prototipo funcional de una prótesis transfemoral, fabricado a partir de los resultados de la sección anterior; la sección 4 presenta los resultados de simulación para el cálculo del torque que se debe aplicar a la articulación de la prótesis para generar el ciclo de marcha. En la sección 5 se detallan las particularidades de la solución, así como un panorama de mejoras que pueden ser aplicadas.

\section{Metodología de diseño}

Los procedimientos de diseño para mecanismos de cuatro barras, parten del análisis de mecanismos planos, tal como se describe ampliamente en (Toussaint, 2003; Radcliffe and Deg, 2003; Narang et al., 2016). De acuerdo con (Nikravesh, 1988), es necesario escoger un sistema de coordenadas con las cuales sea posible describir un sistema mecánico. Ese tipo de coordenadas son las llamadas coordenadas generalizas, definiendo a éstas como un conjunto de coordenadas independientes entre si, capaz de especificar completamente la configuración de un sistema (Haug, 1984; Nikravesh, 1988; Poznyak, 2005).

\subsection{Análisis dinámico de sistemas planares}

La Figura 2 muestra la secuencia de las etapas para el tratamiento dinámico de un sistema multicuerpo de cadena cerrada, que deberá funcionar como la articulación de rodilla. Una vez definida la longitud y conformación de los cuatro eslabones, se prefiere el uso de las coordenadas cartesianas para especificar la distribución y posición del mecanismo cruzado, debido a la simplicidad que representa la derivación de las ecuaciones de movimiento, así como al bajo orden de no linealidades. Por otro lado, los inconvenientes son la cantidad de ejes y de ecuaciones diferenciales de segundo orden (Haug, 1989; Etchechoury,

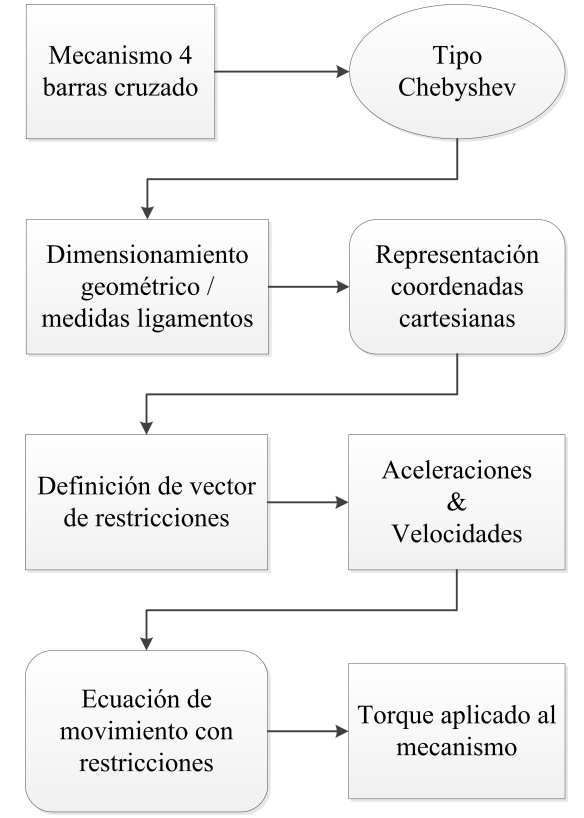

Figura 2: Estrategia aplicada para el diseño de un mecanismo planar, para uso prostético

2001). Al margen de la selección del tipo de coordenadas independientes, se debe tener en cuenta que las características del mecanismo predisponen de restricciones al movimiento, asociadas a un sistema de coordenadas dependientes, las cuales generalmente proporcionan un conjunto de ecuaciones no lineales acopladas, que deben ser resueltas de manera simultánea, mediante un algoritmo análisis númerico (Haug, 1984).

\subsection{Sintesis geométrica de un mecanismo planar}

Las restricciones cinemáticas representan los impedimentos de movimiento relativo a la conexión de los eslabones de una estructura, siendo dependientes solamente de las coordenadas relativas. Por otro lado, las restricciones dinámicas están asociadas a la posición respecto al tiempo, producida por el actuador conectado al mecanismo, que depende de las coordenadas del sistema y del tiempo; entonces, se puede construir el vector de restricciones como sigue:

$$
\boldsymbol{\Psi}(\boldsymbol{r}, t)=\left[\begin{array}{c}
\boldsymbol{\Psi}^{K}(\boldsymbol{r}) \\
\boldsymbol{\Psi}^{D}(\boldsymbol{r}, t)
\end{array}\right]=0
$$

donde $\boldsymbol{\Psi}^{K}$ y $\boldsymbol{\Psi}^{D}$ representan las restricciones cinemáticas y dinámicas; respectivamente, $\boldsymbol{r}$ es el vector de coordenadas cartesianas. Al derivar ambos lados de (1) con respecto al tiempo, se obtiene la expresión para calcular la velocidad del sistema:

$$
\boldsymbol{\Psi}_{r} \dot{\boldsymbol{r}}=-\boldsymbol{\Psi}_{t} \equiv \boldsymbol{v}
$$

De manera similar a la deducción anterior, la derivada respecto al tiempo de ambos lados de (2) permite obtener las aceleraciones del sistema, como sigue:

$$
\boldsymbol{\Psi}_{r} \ddot{\boldsymbol{r}}=-\left(\boldsymbol{\Psi}_{r} \dot{\boldsymbol{r}}\right)_{r} \dot{\boldsymbol{r}}-2 \boldsymbol{\Psi}_{t r} \dot{\boldsymbol{r}}-\boldsymbol{\Psi}_{t t} \equiv \boldsymbol{\gamma}
$$


donde $\boldsymbol{\Psi}_{t r}=\boldsymbol{\Psi}_{r t}$ representa la derivada parcial de la matriz Jacobiana respecto al tiempo, $\boldsymbol{\Psi}_{t t}$ indica la segunda derivada de $\boldsymbol{\Psi}$ respecto al tiempo.

Las ecuaciones que representan la posición, velocidad y aceleración; (1), (2) y (3) respectivamente, sirven de base para definir las ecuaciones de movimiento de sistemas planos con restricciones. De acuerdo a (Haug, 1984), dicha deducción se puede realizar a partir de una ecuación de variación de movimiento, teniendo en cuenta que las fuerzas internas y externas que actúan dentro de un sistema están incluidas en un vector $\boldsymbol{Q}_{i}$.

La expresión de movimiento variacional, originada a partir del principio de trabajo virtual; se expresa como:

$$
\sum_{i=1}^{n b} \delta \boldsymbol{r}_{i}^{\top}\left[\boldsymbol{M}_{i} \ddot{\boldsymbol{r}}_{i}-\boldsymbol{Q}_{i}^{A}\right]-\sum_{i=1}^{n b} \delta \boldsymbol{r}_{i}^{\top} \boldsymbol{Q}_{i}^{C}=0
$$

donde $\boldsymbol{r}_{i}$ representa las coordenadas de posición, $\ddot{\boldsymbol{r}}_{i}$ es la aceleración en coordenadas cartesianas, $\boldsymbol{M}_{i}$ es la matriz diagonal de masa del cuerpo $i$, las expresiones $\boldsymbol{Q}_{i}^{A}$ y $\boldsymbol{Q}_{i}^{C}$ sugieren los vectores compuestos de fuerzas aplicadas y fuerzas de restricciones, respectivamente. En particular, $\boldsymbol{Q}_{i}^{A}$ es el vector de tres elementos (respecto a las coordenadas $M, N$ y $\psi$ ) que representan fuerzas o torques que son aplicados, $n b$ es el número de eslabones del sistema, por otro lado, el enfoque del análisis se centra en los desplazamientos virtuales coincidentes con las restricciones presentes en el mecanismo, por ello el trabajo virtual de las fuerzas de restricción $\boldsymbol{Q}_{i}^{C}$ se iguala a cero.

Empleando el método de los multiplicadores de Lagrange descrito en (Nikravesh, 1988; Haug, 1984); se considera que las fuerzas internas que actúan sobre un cuerpo, pueden ser descritas en función del Jacobiano de restricciones y un vector de incógnitas $\lambda$. De tal manera que la ecuación de movimiento de un sistema de varios eslabones puede ser definida como:

$$
\boldsymbol{M} \ddot{\boldsymbol{r}}+\boldsymbol{\Psi}_{r}^{\top} \boldsymbol{\lambda}=\boldsymbol{Q}^{A}
$$

Las expresiones (1) y (5) representan un sistema de Ecuaciones Diferenciales Algebraicas (en adelante EDA) de índice 3 , la resolución de este tipo de configuraciones resulta compleja (Haug, 1984); por lo que es recomendable derivar la ecuación de restricción (1) para reducir el índice, según se sugiere en (Etchechoury, 2001). El resultado de se agrega a (5), ambas expresiones representan un sistema de Ecuaciones Diferenciales Ordinarias (en adelante EDO) o un sistema EDA de índuce 1, como se muestra a continuación:

$$
\left[\begin{array}{cc}
\boldsymbol{M} & \boldsymbol{\Psi}_{r}^{\top} \\
\boldsymbol{\Psi}_{r} & 0
\end{array}\right]\left[\begin{array}{c}
\ddot{\boldsymbol{r}} \\
\lambda
\end{array}\right]=\left[\begin{array}{c}
\boldsymbol{Q}^{A} \\
\boldsymbol{\gamma}
\end{array}\right]
$$

La integración numérica de una EDA de índice 1, como la presentada en (6); tiene problemas en la solución, debido a la inestabilidad que se puede asociar al uso de la segunda derivada de la ecuación de restricción, por la información que se pierde al realizar las derivadas.

Una alternativa de solución es utilizar algoritmos de integración eficientes para calcular la solución de acuerdo a requisitos determinados, como los problemas de primer orden de valor

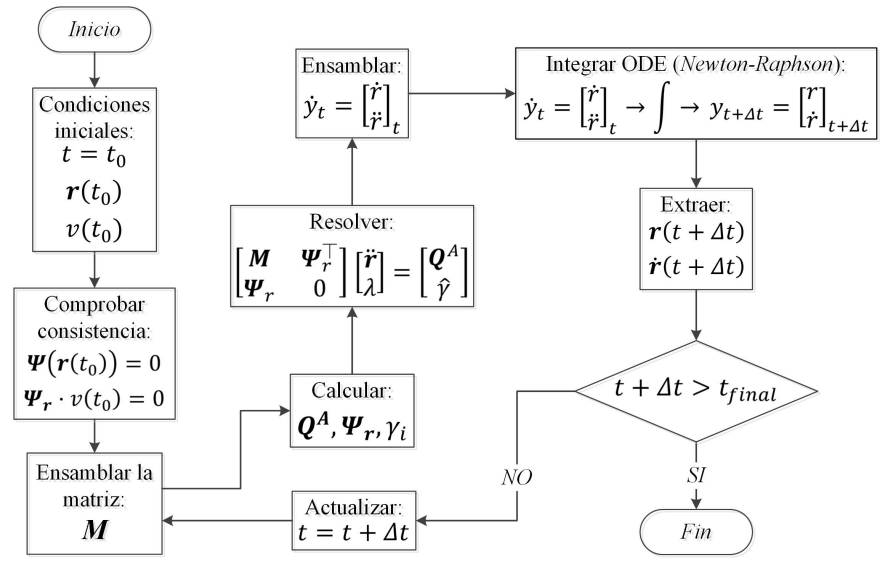

Figura 3: Diagrama de bloques para evaluar las violaciones de restricciones del problema cinamático de posición, velocidad y aceleración.

inicial que, permite transformar sistemas de ecuaciones de movimiento de tipo EDA en sistemas de primer orden de valor inicial, de acuerdo a la siguiente expresión:

$$
\dot{y}_{t}=\left\{\dot{r}^{\top}, \ddot{r}^{\top}\right\}_{t}^{\top} \rightarrow \int \rightarrow y_{t+\Delta t}=\left\{r^{\top}, \dot{r}^{\top}\right\}_{t+\Delta t}^{\top}
$$

donde $y$ es el vector de coordenadas de posición, $\dot{y}$ es el vector de velocidades y $\ddot{y}$ representa el vector de aceleraciones. El procedimiento de integración considera que los vectores de posición y velocidad en un tiempo posterior $t+\Delta t$ determinan las aceleraciones y velocidades generalizadas en el tiempo actual $t$.

Por lo anterior, se propone utilizar el algoritmo NewtonRaphson para integrar numéricamente las velocidades y aceleraciones para el paso de tiempo dado; con esto se puede resolver la ecuación (7); que a su vez satisface el conjunto de ecuaciones no lineales que produce la condición de restricción $\boldsymbol{\Psi}(\boldsymbol{r}, t)=0$.

El principal problema para integrar la expresión matricial (6) es la llamada estabilización de violación de restricciones cinemáticas, de acuerdo a (Haug, 1989), dado que la restricción de aceleración, definida como $\boldsymbol{\Psi}_{r} \ddot{\boldsymbol{r}}=0$; puede ser inestable. Por ello es posible utilizar el método de estabilización de Baumgarte (Haug, 1989), el cual radica en determinar una expresión nueva de aceleración $\hat{\gamma}$ a partir de (3) y sustituirlo en (6), como sigue:

$$
\Psi_{r} \ddot{r}=\gamma-2 \alpha\left(\Psi_{r} \dot{r}+\Psi_{t}\right)-\beta^{2} \Psi \equiv \hat{\gamma}
$$

donde $\alpha$ y $\beta$ son constantes positivas que caracterizan el lazo de retroalimentación durante la infracción de las restricciones de posición, velocidad y/o aceleración. La selección de las constantes anteriores no asegura la convergencia del método de integración, sino del integrador númerico. De acuerdo a (Haug, 1989) se sugiere utilizar a $\alpha>0, \beta \neq 0$ y en ambos casos $\alpha=\beta \leq 10$; para que sea posible estabilizar la expresión (8) utilizando el algoritmo de integración directa de una EDO (Haug, 1984, 1989) que, se resume en la Figura 3.

Para el análisis de la dinámica inversa del mecanismo de cuatro barras cruzadas de la Figura 4, se identifican los eslabones móviles; centros de masa y ángulos de orientación, con lo cual, 
es posible definir el vector de coordenadas cartesianas, que describe la configuración del mecanismo a partir de la siguiente expresión:

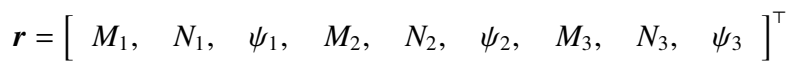

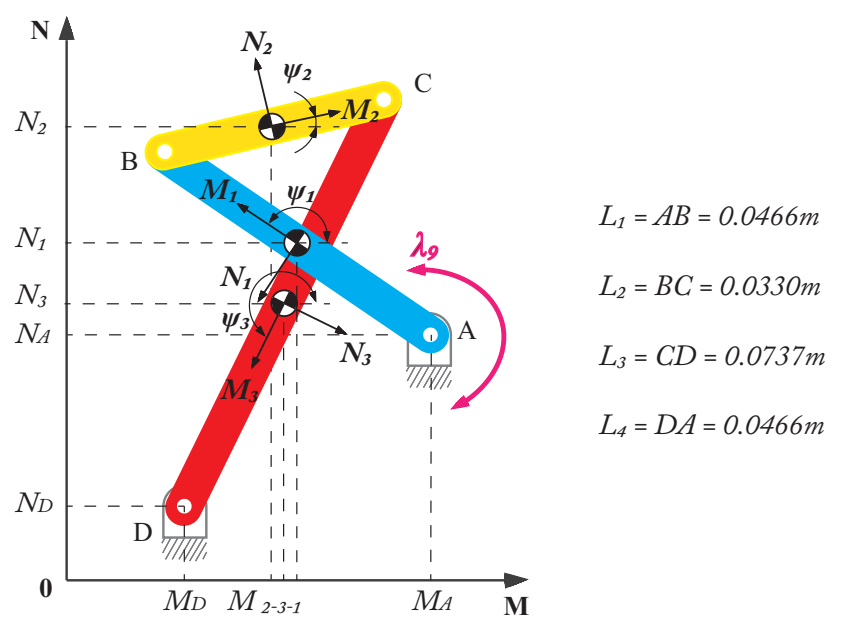

Figura 4: Mecanismo de cuatro barras cruzado representado en coordenadas cartesianas

En (10) se escribe el vector de restricciones. Las primeras dos líneas están asociadas a las restricciones cinemáticas absolutas de posición para el punto A; en ellas, las coordenadas del eslabón $\overline{A B}$, definido como $p_{1}=\left[M_{1}, N_{1}\right]$ está vinculada al punto fijo $A=\left[M_{A}, N_{A}\right]$. Lo anterior se muestra en la figura 5(a). De la tercera a la sexta línea de (10) describen las restricciones cinemáticas para las juntas de revoluta de los puntos $\mathrm{B}$ y C. Esto significa que la posición del punto B es el mismo, tanto para la barra $\overline{A B}$ como para $\overline{B C}$ mostrado en la Figura 5(b).

La consideración anterior se toma para el punto $C$ que, es el punto de unión de las barras $\overline{B C}$ y $\overline{C D}$, en la figura 5(c) se identifican las restricciones para la junta $C$. El punto fijo D está representado por la séptima y octava líneas de (10) que indican las restricciones cinemáticas absolutas de posición, la gráfica de los vectores de dicho punto es presentada en la Figura 5(d). La novena línea es la única restricción dinámica, considerando que el mecanismo estudiado solamente tiene un grado de libertad. En dicha línea, se tiene que $\psi_{1}$ gira a una velocidad angular constante $\omega_{4 b}$; y el término $\psi_{1_{0}}$ indica la posición inicial de $\psi_{1}$.

$$
\boldsymbol{\Psi}=\left[\begin{array}{c}
M_{1}-\frac{L_{1}}{2} \cos \left(\psi_{1}\right)-M_{A} \\
N_{1}-\frac{L_{1}}{2} \operatorname{sen}\left(\psi_{1}\right)-N_{A} \\
M_{1}+\frac{L_{1}}{2} \cos \left(\psi_{1}\right)-M_{2}+\frac{L_{2}}{2} \cos \left(\psi_{2}\right) \\
N_{1}+\frac{L_{1}}{2} \operatorname{sen}\left(\psi_{1}\right)-N_{2}+\frac{L_{2}}{2} \operatorname{sen}\left(\psi_{2}\right) \\
M_{2}+\frac{L_{2}}{2} \cos \left(\psi_{2}\right)-M_{3}+\frac{L_{3}}{2} \cos \left(\psi_{3}\right) \\
N_{2}+\frac{L_{2}}{2} \operatorname{sen}\left(\psi_{2}\right)-N_{3}+\frac{L_{3}}{2} \operatorname{sen}\left(\psi_{3}\right) \\
M_{3}+\frac{L_{3}}{2} \cos \left(\psi_{3}\right)-M_{D} \\
N_{3}+\frac{L_{3}}{2} \operatorname{sen}\left(\psi_{3}\right)-N_{D} \\
\psi_{1}-\psi_{1_{0}}-\omega_{4 b}(t)
\end{array}\right]=0
$$

El siguiente paso es formar los vectores que permitan calcular la velocidad (2) y las aceleraciones (3), ya que uno de los planteamientos del problema del análisis del mecanismo de cuatro barras se tiene que $\dot{\omega}_{4 b}=0$. El vector $\Psi_{t}$ queda como sigue:

$$
\boldsymbol{\Psi}_{t}=\left[\begin{array}{lllllllll}
0 & 0 & 0 & 0 & 0 & 0 & 0 & 0 & -\omega_{4 b}
\end{array}\right]^{\top}
$$

Por otro lado, el vector $\Psi_{t t}$ es un vector nulo de dimensión $[1 \times 9]$ y $\Psi_{r t}$ es una matriz nula de dimensión [9 $\times 9$ ]. El Jacobiano del problema se define en la siguiente ecuación:

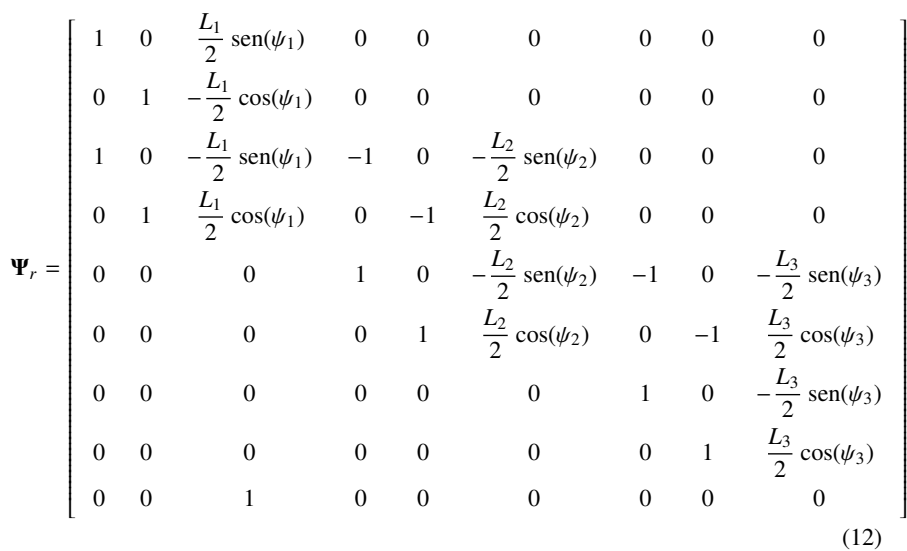

Con lo anterior, la solución de (2) se muestra en la siguiente 


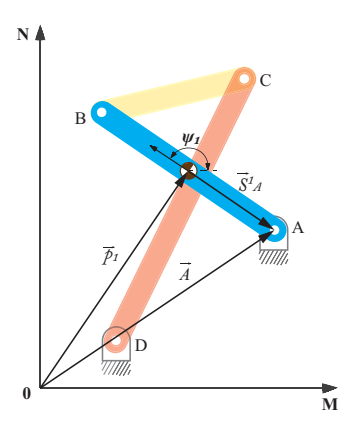

(a) Punto A

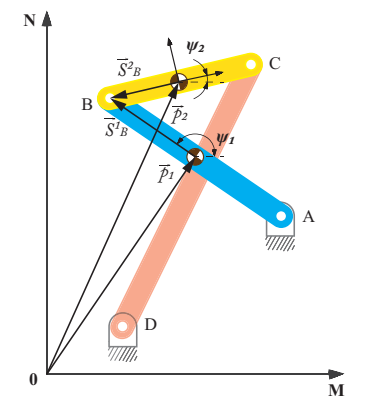

(b) Punto B

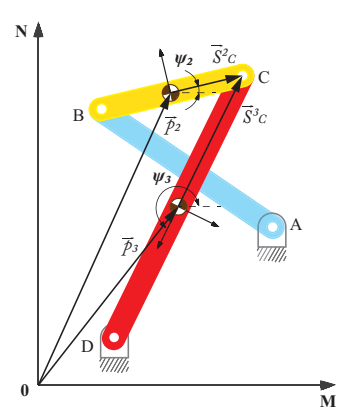

(c) Punto C

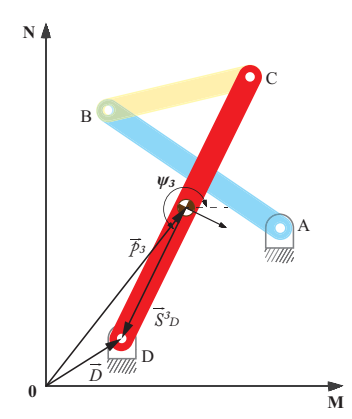

(d) Punto D

Figura 5: Restricciones absolutas de distancia, para determinar la posición de las juntas de revoluta.

ecuación:

$$
\gamma=\left[\begin{array}{c}
-\frac{L_{1}}{2} \cos \left(\psi_{1}\right) \dot{\psi}_{1}^{2} \\
-\frac{L_{1}}{2} \operatorname{sen}\left(\psi_{1}\right) \dot{\psi}_{1}^{2} \\
\frac{L_{1}}{2} \cos \left(\psi_{1}\right) \dot{\psi}_{1}^{2}+\frac{L_{2}}{2} \cos \left(\psi_{2}\right) \dot{\psi}_{2}^{2} \\
\frac{L_{1}}{2} \operatorname{sen}\left(\psi_{1}\right) \dot{\psi}_{1}^{2}+\frac{L_{2}}{2} \operatorname{sen}\left(\psi_{2}\right) \dot{\psi}_{2}^{2} \\
\frac{L_{2}}{2} \cos \left(\psi_{2}\right) \dot{\psi}_{2}^{2}+\frac{L_{3}}{2} \cos \left(\psi_{3}\right) \dot{\psi}_{3}^{2} \\
\frac{L_{2}}{2} \operatorname{sen}\left(\psi_{2}\right) \dot{\psi}_{2}^{2}+\frac{L_{3}}{2} \operatorname{sen}\left(\psi_{3}\right) \dot{\psi}_{3}^{2} \\
\frac{L_{3}}{2} \cos \left(\psi_{3}\right) \dot{\psi}_{3}^{2} \\
\frac{L_{3}}{2} \operatorname{sen}\left(\psi_{3}\right) \dot{\psi}_{3}^{2} \\
0
\end{array}\right]=0
$$

Queda por definir $\boldsymbol{M} \in \mathbb{R}^{9 \times 9}$ que, es la matriz diagonal de masa, cuyos elementos principales se muestran en la siguiente expresión:

$$
\boldsymbol{M}=\operatorname{diag}\left\{\begin{array}{lllllllll}
m_{1} & m_{1} & J_{1} & m_{2} & m_{2} & J_{2} & m_{3} & m_{3} & J_{3}
\end{array}\right\}
$$

donde $m_{i}$ indica la masa del eslabón $i$ y $J_{i}$ representa el momento de inercia alrededor del centro de gravedad del eslabón $i$; con $i=1,2,3$.

Además, se define el vector de fuerzas generalizadas $Q^{A}$, cuyos elementos no nulos son el peso de las tres barras en dirección negativa de $y$, debido a la acción de la fuera de gravedad $g$ :

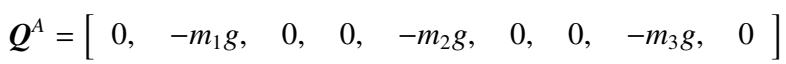

Una vez que se han definido las matrices, es posible expandir (6), como se muestra debajo; el término $\ddot{\boldsymbol{r}}$ no es conocido explícitamente, pero puede ser calculado despejando de (16):

$$
\begin{aligned}
\boldsymbol{M} \ddot{\boldsymbol{r}}+\boldsymbol{\Psi}_{r}^{\top} \boldsymbol{\lambda} & =\boldsymbol{Q}^{A} \\
\boldsymbol{\Psi}_{r} \ddot{\boldsymbol{r}} & =\gamma
\end{aligned}
$$

La única incógnita de (16) es $\lambda$. Por tanto si el Jacobiano es no singular; se puede calcular $\lambda$ como sigue:

$$
\lambda=\left(\Psi_{r}^{\top}\right)^{-1}\left(Q^{A}-M \ddot{r}\right)
$$

El propio análisis de (6) permite comprender el papel de los Multiplicadores de Lagrange. La expresión (18) muestra nueve ecuaciones relativas a la sumatoria de fuerzas (masa por aceleración) y a la sumatoria de momentos (momento de inercia por la aceleración angular), que corresponden a la expansión de la primera línea de (16).

$$
\begin{aligned}
m_{1} \ddot{M}_{1}+\lambda_{1}+\lambda_{3} & =0 \\
m_{1} \ddot{N}_{1}-\lambda_{2}+\lambda_{4}+m_{1} g & =0 \\
J_{1} \ddot{\psi}_{1}+\frac{L_{1} \lambda_{1} \operatorname{sen}\left(\psi_{1}\right)}{2}-\frac{L_{1} \lambda_{2} \cos \left(\psi_{1}\right)}{2}-\frac{L_{1} \lambda_{3} \operatorname{sen}\left(\psi_{1}\right)}{2}+\frac{L_{1} \lambda_{4} \cos \left(\psi_{1}\right)}{2}+\lambda_{9} & =0 \\
m_{2} \ddot{M}_{2}+\lambda_{3}+\lambda_{5} & =0 \\
m_{2} \ddot{N}_{2}-\lambda_{4}+\lambda_{6}+m_{2} g & =0 \\
J_{2} \ddot{\psi}_{2}-\frac{L_{2} \lambda_{3} \operatorname{sen}\left(\psi_{2}\right)}{2}+\frac{L_{2} \lambda_{4} \cos \left(\psi_{2}\right)}{2}-\frac{L_{2} \lambda_{5} \operatorname{sen}\left(\psi_{2}\right)}{2}+\frac{L_{2} \lambda_{6} \cos \left(\psi_{2}\right)}{2} & =0 \\
m_{3} \ddot{M}_{3}+\lambda_{5}+\lambda_{7} & =0 \\
m_{3} \ddot{N}_{3}-\lambda_{6}+\lambda_{8}+m_{3} g & =0 \\
J_{3} \ddot{\psi}_{3}-\frac{L_{3} \lambda_{5} \operatorname{sen}\left(\psi_{3}\right)}{2}+\frac{L_{3} \lambda_{6} \cos \left(\psi_{3}\right)}{2}-\frac{L_{3} \lambda_{7} \operatorname{sen}\left(\psi_{3}\right)}{2}+\frac{L_{3} \lambda_{8} \cos \left(\psi_{3}\right)}{2} & =0
\end{aligned}
$$

Las siguientes nueve expresiones corresponden a la expansión de la restricción de aceleración de la segunda línea de (16)

$$
\begin{aligned}
\ddot{M}_{1}-\frac{L_{1} \ddot{\psi}_{1} \operatorname{sen}\left(\psi_{1}\right)}{2}+\frac{L_{1} \dot{\psi}_{1}^{2} \cos \left(\psi_{1}\right)}{2}=0 \\
\ddot{N}_{1}-\frac{L_{1} \ddot{\psi}_{1} \cos \left(\psi_{1}\right)}{2}+\frac{L_{1} \dot{\psi}_{1}^{2} \operatorname{sen}\left(\psi_{1}\right)}{2}=0 \\
\ddot{M}_{1}-\frac{L_{1} \ddot{\psi}_{1} \operatorname{sen}\left(\psi_{1}\right)}{2}-\ddot{M}_{2}-\frac{L_{2} \ddot{\psi}_{2} \operatorname{sen}\left(\psi_{2}\right)}{2}-\frac{L_{1} \dot{\psi}_{1}^{2} \cos \left(\psi_{1}\right)}{2}+\frac{L_{2} \dot{\psi}_{2}^{2} \cos \left(\psi_{3}\right)}{2}=0 \\
\ddot{N}_{1}-\frac{L_{1} \ddot{\psi}_{1} \cos \left(\psi_{1}\right)}{2}-\ddot{N}_{2}-\frac{L_{2} \ddot{\psi}_{2} \cos \left(\psi_{2}\right)}{2}-\frac{L_{1} \dot{\psi}_{1}^{2} \operatorname{sen}\left(\psi_{1}\right)}{2}+\frac{L_{2} \dot{\psi}_{2}^{2} \operatorname{sen}\left(\psi_{3}\right)}{2}=0 \\
\ddot{M}_{2}-\frac{L_{2} \ddot{\psi}_{2} \operatorname{sen}\left(\psi_{2}\right)}{2}-\ddot{M}_{3}-\frac{L_{3} \ddot{\psi}_{3} \operatorname{sen}\left(\psi_{3}\right)}{2}+\frac{L_{2} \dot{\psi}_{2}^{2} \cos \left(\psi_{2}\right)}{2}+\frac{L_{3} \dot{\psi}_{3}^{2} \cos \left(\psi_{3}\right)}{2}=0 \\
\ddot{N}_{2}-\frac{L_{2} \ddot{\psi}_{2} \cos \left(\psi_{2}\right)}{2}-\ddot{N}_{3}-\frac{L_{3} \ddot{\psi}_{3} \cos \left(\psi_{3}\right)}{2}+\frac{L_{2} \dot{\psi}_{2}^{2} \operatorname{sen}\left(\psi_{2}\right)}{2}+\frac{L_{3} \dot{\psi}_{3}^{2} \operatorname{sen}\left(\psi_{3}\right)}{2}=0 \\
\ddot{M}_{3}-\frac{L_{3} \ddot{\psi}_{3} \operatorname{sen}\left(\psi_{3}\right)}{2}+\frac{L_{3} \dot{\psi}_{3}^{2} \cos \left(\psi_{3}\right)}{2}=0 \\
\ddot{N}_{3}-\frac{L_{3} \ddot{\psi}_{3} \cos \left(\psi_{3}\right)}{2}+\frac{L_{3} \dot{\psi}_{3}^{2} \operatorname{sen}\left(\psi_{3}\right)}{2}=0
\end{aligned}
$$

De acuerdo a la distribución de los términos de (18), la tres primeras líneas están relacionadas con el eslabón $\overline{A B}$, el cual es el encargado de hacer rotar al mecanismo. En la tercera línea de (18) se puede comprobar que el único término aislado es $\lambda_{9}$, lo que significa que el torque que se debe imprimir en sentido 
contrario sobre el eslabón $\overline{A B}$ para lograr que el mecanismo gire a una velocidad constante.

La propuesta de solución radica en calcular el torque del actuador que sea adecuado para movilizar el mecanismo cruzado, a partir de la información geométrica e inercial de los eslabones del mecanismo; esto es la base del planteamiento de un problema de dinámica inversa. Por otro lado, si el torque es conocido, así como las condiciones iniciales de los grados de libertad, es posible calcular la respuesta en el tiempo (posición, velocidad y aceleración) de un mecanismo, a través de la simulación dinámica o la dinámica directa. Si los resultados entre ambos problemas dinámicos son coherentes y estables, es posible confirmar la validación de la síntesis geométrica de un mecanismo de cadena cerrada como el que se discute en esta sección.

\section{Validación mediante uso de prototipo}

Para la validación del torque se ha construido un prototipo con características suficientes para aplicar un precedimiento de control robusto con un observador de estados que solo requiere un modelo generalizado de segundo orden del dispositivo prostético, cuyos parámetros permiten activar una máquina de estados finita (en adelante MEF), para el seguimiento de una trayectoria de referencia, similar a la de una rodilla sana durante el ciclo de marcha. La Figura 6 simplifica la etapa de control automático para una prótesis transfemoral activa.

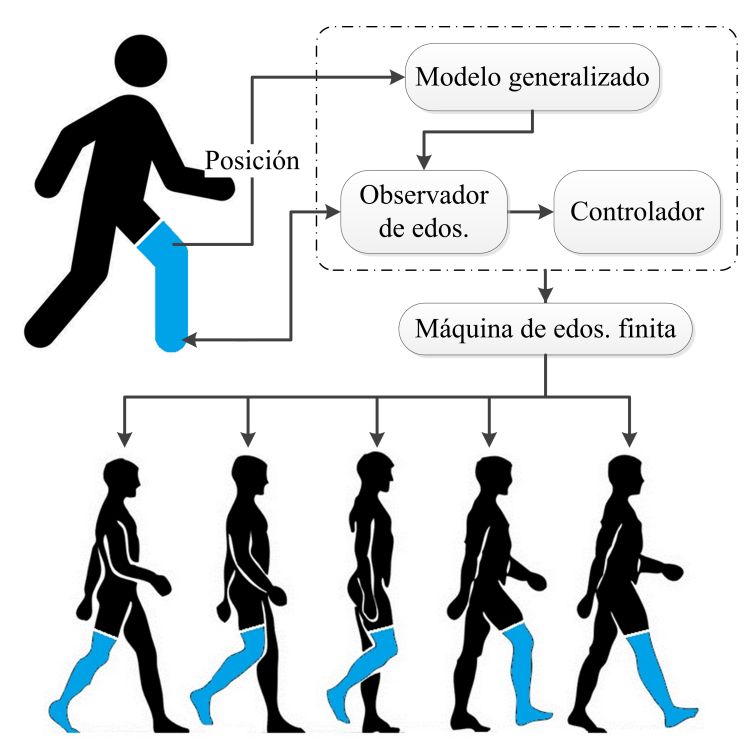

Figura 6: La estrategia de control implementada para la prótesis, así como las secuencias de posturas adecuadas para reproducir la marcha humana

Una MEF es una conceptualización informática que indica el comportamiento de un sistema activo, que está conformado por:

- Estados: son las condiciones en las que se encuentra sistema, subsistema u objeto en algún instante determinado.

- Transiciones: determinan la forma bajo la cual cada estado transita a otro estado, en correspondencia a un evento.
Tabla 1: Las ocho fases del ciclo de marcha se pueden representan en seis estados discretos

\begin{tabular}{|l|l|}
\hline Fases ciclo de marcha & Estados MEF \\
\hline \hline Contacto Inicial & Cont_Inicial (F1) \\
\hline Respuesta de carga & Resp_Carga (F2) \\
\hline Apoyo medio & Apoyo_Med (F3) \\
\cline { 1 - 2 } Apoyo final & Apoyo_Fin (F4) \\
\cline { 1 - 2 } Pre-balanceo & \multirow{2}{*}{ Balance_1 (F5) } \\
\cline { 1 - 2 } Balanceo inicial & \multirow{2}{*}{ Balance_2 (F6) } \\
\cline { 1 - 2 } Balanceo medio & \\
\cline { 1 - 2 } Balanceo final &
\end{tabular}

- Evento: Son las condiciones necesarias que producen un cambio dentro del sistema, lo que se traduce en un cambio de estado.

Con esta definición, los estados de la MEF para el sistema prostético se determinan a partir de las ocho fases del ciclo de marcha; y se presentan en la Tabla 1.

\section{Resultados}

La mayoría de los mecanismos de cuatro barras que están diseñados como articulación de rodilla, están dispuestos con la forma geométrica tradicional. Sin embargo, de acuerdo a los trabajos de investigación en los cuales están involucrados este tipo de mecanismos, el centro instantáneo de rotación está fuera de la geometría del mecanismo en cualquier posición en que sea movido; en el caso de este trabajo se considera un mecanismo de cuatro barras cruzado que es una variación del mecanismo de Chebyshev que busca principalmente convertir el movimiento rotacional de una de las barras que está actuada, en un movimiento lineal de un punto central ubicado en uno de los eslabones. La disposición cinemática de esta configuración, es coincidente con la ubicación de dos ligamentos importantes involucrados en flexiones en el plano sagital, esto permite aprovechar la rotación del eslabón en donde se ubica la trayectoria lineal; además el centro instantáneo de rotación tiene una traslación similar al de una rodilla humana sana. Los resultados de este trabajo se presentan en dos vertientes; la primera y más relevante como la solución de un algoritmo que permita realizar una simulación en Matlab para corroborar que la síntesis geométrica desarrollada es adecuada para generar el movimiento de la rodilla durante el ciclo de marcha, a partir de un torque similar al observado en una extremidad sana. Como un segundo nivel de contribución, se presenta el resultado fenomenológico de un prototipo mínimo viable, a partir de un controlador robusto gobernado por un autómata finito.

\subsection{Torque requerido para generar el ciclo de marcha}

Considerando el rango natural de movimiento de una articulación rodilla sana y los valores reportados correspondientes al ciclo de marcha en (Perry, 1992), se tiene que son valores positivos; en otras palabras, solamente interviene el movimiento de flexión; ya que la posición de referencia de la rodilla se 
sitúa en alargamiento; considerando la definición de extensión como: el alejamiento de la cara posterior de la pierna de la cara posterior del muslo.

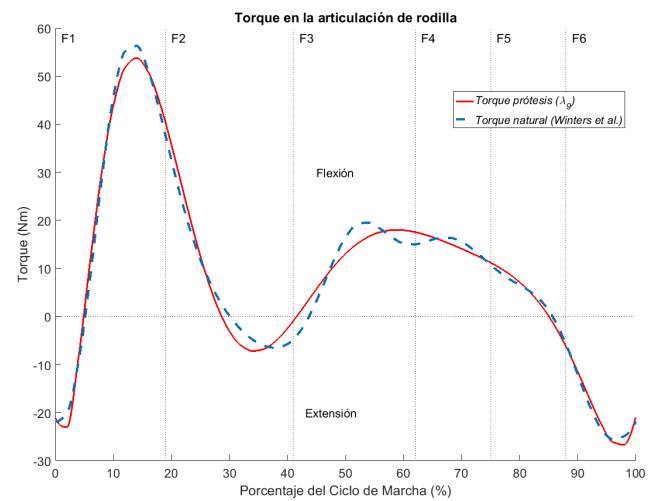

Figura 7: Torque en la articulación A necesario para mover el mecanismo calculado mediante dinámica inversa, comparado con el torque natural de la rodilla de acuerdo a (Winter, 2009)

La síntesis descrita en la Sección 2 propone una solución de la dinámica inversa para un mecanismo cruzado que representa la rodilla, mediante la aplicación de ecuaciones diferenciales algebráicas. Esta metodología fue implementada en Matlab, considerando como principales datos: 105 kilogramos como la masa del usuario de la prótesis, un rango de movimiento de la rodilla entre 0 a $60^{\circ}$ y las longitudes de los ligamentos cruzados de un adulto de aproximadamente 1,70 metros de estatura. El principal resultado obtenido fue el torque que se debe aplicar al eslabón $\overline{A B}$ durante el ciclo de marcha, de tal manera que se pueda conseguir la postura necesaria para cada fase del ciclo de marcha natural. En la Figura 7 se muestra el torque que debe producir la rodilla en marcha (en línea azul discontinua), de acuerdo a (Winter, 2009). Comparamos lo anterior con nuestro resultado de dinámica inversa (en línea roja continua) identificando los estados discretos de la máquina de estado, identificados como $F_{i}$, con $i=1,2, . .6$.

En la Figura 7 se muestra que nuestro resultado de simulación para el torque máximo es de $52 \mathrm{Nm}$ en la primera fase, un valor muy carcano a los $55 \mathrm{Nm}$ producidos en una rodilla sana a causa de la dinámica de la fase respuesta de carga (Perry, 1992). Por otro lado, se puede advertir diferencias entre el torque natural y el torque próstetico en las fases 2 y 3; que pueden obedecer a los cambios de dirección del actuador de la prótesis. En el resto de las fases se puede apreciar que la rodilla prostética puede reproducir las características de fuerza de una rodilla sana.

Para comparar el desempeño del análisis de dinámica inversa, se reprodujo un algoritmo de dinámica directa, que pretende verificar la posición, velocidad y aceleración de los centros de masa de los eslabones móviles del mecanismo de cuatro barras cruzado, que demuestra la factibilidad de las condiciones cinemáticas para un movimiento tipo pendular de la rodilla prostética, durante 0,4 segundos, que es el tiempo de la simulación en el que se satisfacen las 6 fases de la MEF. El resultado se puede consultar en la Figura 8.
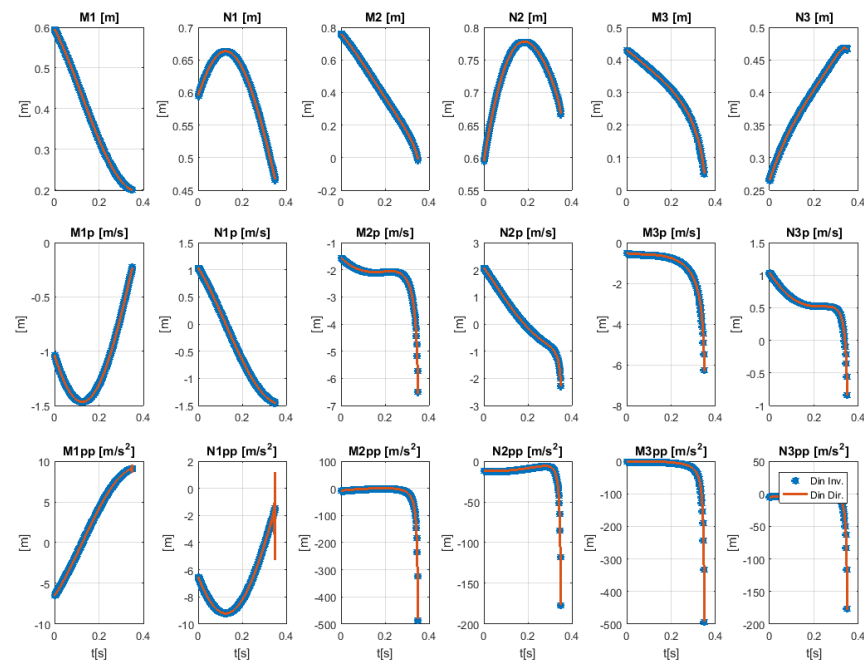

Figura 8: Resultados y comparación del estudio cinemático del mecanimo de cuatro barras cruzado, durante una trayectoria de recorrido, comparando posición, velocidad y aceleración

\subsection{Ejecución del ciclo de marcha prostética}

La demostración de viabilidad mecánica se muestra en la Figura 9, en donde se identifican los fotogramas de los seis estados discretos de la MEF, que reproduce el ciclo de marcha.

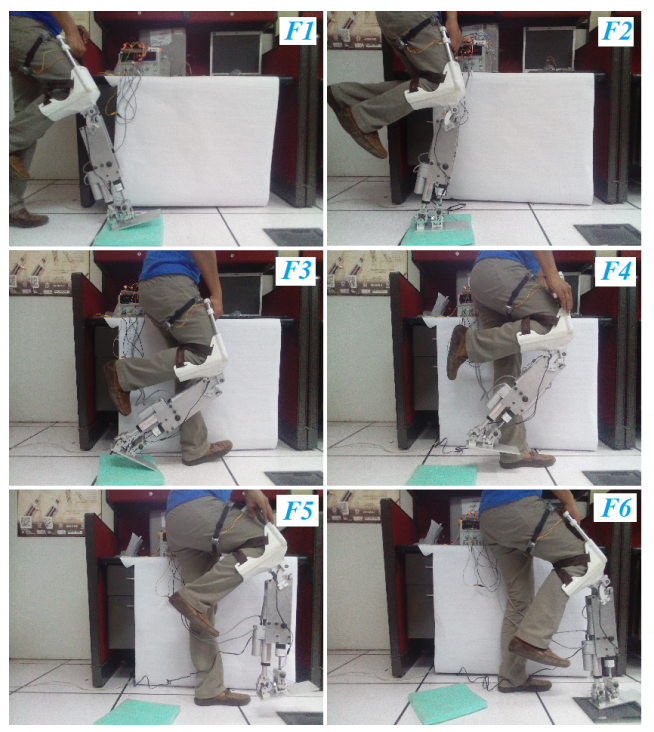

Figura 9: Ejecución del ciclo de marcha prostética con el prototipo para realizar una zancada comenzando desde la base color verde y terminando en la base color negra.

La experimentación en el prototipo mínimo viable permitió valorar las condiciones de diseño mecánico que aseguren la correcta transferencia de la masa del usuario que debe ser balanceada para conseguir el avance de una zancada.

\section{Conclusión}

Los resultados de este trabajo de investigación permitieron proponer un mecanismo significativamente eficiente para las 
particularidades del movimiento de una rodilla sobre el plano sagital. Por ello se confirma la hipótesis que relaciona los mecanismos planares para el uso en sistemas biomecánicos y de de rehabilitación para movimientos que se han visto afectados por enfermedades o amputaciones.

El diseño mecánico ha permitido realizar un prototipo y comprobar el rango de movimiento adecuado para las articulaciones de rodilla y tobillo. A partir de esto, se debe continuar con la fabricación en el material final, con el cual se realizó el análisis estructural; a partir de la selección de los actuadores, y piezas mecánicas que forman el actuador elástico en serie, se debe construir este actuador e integrar a la estructura prostética.

Una vez que se ha validado el diseño mecánico a través de la simulación de la dinámica inversa, queda como trabajo a futuro mejorar la plataforma de prueba así como la integración con el esquema de control automático.

\section{English Summary}

\section{Design of a knee joint based on four crossed bar chebys- hev Type polycentric mechanism.}

\begin{abstract}
The present work presents the results of the design of a knee joint for prostheses, exoskeletons and bipedal robots, made from the geometric synthesis for planar mechanisms. Taking advantage of the anatomical advantages provided by the mechanisms of four bars to reproduce the movement of the knee on the sagittal axis, in the gait cycle; a configuration of crossed bars is presented, which mimics the performance of the crossed ligaments of the knee. According to the results obtained, it was possible to construct a minimum prototype of an active transfemoral prosthesis to perform the complete cycle of gait, which is adequate to the parameters of locomotion in adults.
\end{abstract}

\section{Keywords:}

Active transfemoral prosthesis, 4-Bars mechanism, Chebyshev mechanism, Geometrical synthesis, Biomedical devices.

\section{Agradecimientos}

Este trabajo ha sido realizado gracias al apoyo de la UPIITA, así como el financiamiento de la SIP-IPN con número de registro 20194990 y 20194994.

\section{Referencias}

Etchechoury, M. d. R., 2001. Sistemas diferenciales-algebraicos: aplicaciones a perturbación singular y control. Ph.D. thesis, Facultad de Ciencias Exactas.

Gini, G., Scarfogliero, U., Folgheraiter, M., 2007. Human-oriented biped robot design: Insights into the development of a truly anthropomorphic leg. In: Proceedings 2007 IEEE International Conference on Robotics and Automation. IEEE, pp. 2910-2915.

Hamill, J., Knutzen, K. M., 2006. Biomechanical basis of human movement. Lippincott Williams \& Wilkins.

Haug, E. J., 1984. Computer aided analysis and optimization of mechanical system dynamics. Springer-Verlag Berlin Heidelberg.

Haug, E. J., 1989. Computer aided kinematics and dynamics of mechanical systems. Vol. 1. Allyn and Bacon Boston.

Herr, H., Wilkenfeld, A., 2003. User-adaptive control of a magnetorheological prosthetic knee. Industrial Robot: An International Journal 30 (1), 42-55.

Kempe, A. B., 1875. On a general method of describing plane curves of the nth degree by linkwork. Proceedings of the London Mathematical Society 1 (1), 213-216.

Kempe, A. B., 1877. How to draw a straight line: a lecture on linkages. Macmillan and Company.

Martinez, R. C., Avitia, R. L., Bravo, M. E., Carranza, M. A. R., 2014. A low cost design of powered ankle-knee prosthesis for lower limb amputeespreliminary results. In: BIODEVICES. pp. 253-258.

Miller, L. A., Childress, D. S., may 2005. Problems associated with the use of inverse dynamics in prosthetic applications: An example using a polycentric prosthetic knee. Robotica 23 (3), 329-335.

DOI: $10.1017 / \mathrm{s} 0263574704001353$

Narang, Y. S., Arelekatti, V. N. M., Winter, A. G., jul 2016. The effects of prosthesis inertial properties on prosthetic knee moment and hip energetics required to achieve able-bodied kinematics. IEEE Trans. Neural Syst. Rehabil. Eng. 24 (7), 754-763.

DOI: $10.1109 /$ tnsre.2015.2455054

Nikravesh, P. E., 1988. Computer-aided analysis of mechanical systems. Prentice-Hall, Inc.

Perry, J., 1992. Gait Analysis: Normal and Pathological Function. Slack Incorporated.

Poliakov, O. M., Lazarev, V. B., Chepenyuk, O. O., 2013. Transfemoral prosthesis with polycentric knee mechanism: Design, kinematics, dynamics and control strategy. Journal of Rehabilitation Robotics 1 (2), 109-123.

Poznyak, A., 2005. Modelado matemático de los sistemas mecánicos, eléctricos y electromecánicos.

Radcliffe, C. W., Deg, M., 2003. Biomechanics of knee stability control with four-bar prosthetic knees. In: Proc. ISPO Australia Annual Meeting.

Ranzani, R., 2014. Adaptive human model-based control for active knee prosthetics. Master's thesis, ETH Zürich.

Toussaint, G., 2003. Simple proofs of a geometric property of four-bar linkages. The American mathematical monthly 110 (6), 482.

Winter, D. A., 2009. Biomechanics and motor control of human movement. John Wiley \& Sons.

Xydas, E. G., aug 2014. Synthesis and analysis of a chebyschev's straight line four-bar linkage for generating a minimum jerk velocity profile. In: Volume 5A: 38th Mechanisms and Robotics Conference. ASME International. DOI: $10.1115 / \operatorname{det} c 2014-35510$ 